\title{
Diurnal Variation of Urinary Fabry Disease Biomarkers during Enzyme Replacement Therapy Cycles
}

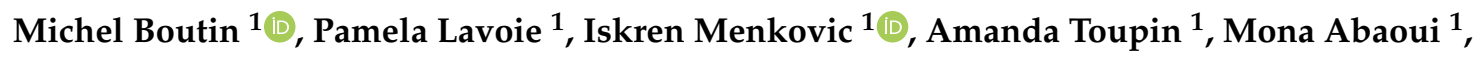 \\ Maha Elidrissi-Elawad ${ }^{1}$, Marie-Françoise Arthus ${ }^{2}$, Carole Fortier ${ }^{2}$, Claudia Ménard ${ }^{2}$, \\ Bruno Maranda ${ }^{1}$, Daniel G. Bichet ${ }^{2,3}$ and Christiane Auray-Blais ${ }^{1, *}$ \\ 1 CIUSSS de l'Estrie-CHUS, Université de Sherbrooke, Centre de Recherche-CHUS, 3001, 12th Avenue North, \\ Sherbrooke, QC J1H 5N4, Canada; Michel.Boutin2@USherbrooke.ca (M.B.); \\ pamela.lavoie@usherbrooke.ca (P.L.); Iskren.Menkovic@USherbrooke.ca (I.M.); \\ amanda.toupin@usherbrooke.ca (A.T.); mona.abaoui@usherbrooke.ca (M.A.); \\ Maha.Elidrissi.Elawad@USherbrooke.ca (M.E.-E.); bruno.maranda@usherbrooke.ca (B.M.) \\ 2 CIUSSS du Nord-de-l'Île-de-Montréal, Hôpital du Sacré-Coeur de Montréal, Clinical Research Unit, \\ 5400 Gouin Blvd West, Montreal, QC H4J 1C5, Canada; marie-francoise.arthus@umontreal.ca (M.-F.A.); \\ C-Fortier@crhsc.rtss.qc.ca (C.F.); claudia.menard@crhsc.rtss.qc.ca (C.M.); \\ daniel.bichet@umontreal.ca (D.G.B.) \\ 3 Department of Medicine Pharmacology and Physiology, Université de Montréal, \\ 2900 Édouard-Montpetit Blvd, Montreal, QC H3T 1J4, Canada \\ * Correspondence: christiane.auray-blais@usherbrooke.ca; \\ Tel.: +1-819-346-1110 (ext. 14706); Fax: +1-819-564-5217
}

Received: 24 July 2020; Accepted: 21 August 2020; Published: 25 August 2020

\begin{abstract}
Fabry disease is an X-linked lysosomal storage disorder caused by mutations in the GLA gene encoding the $\alpha$-galactosidase A enzyme. This enzyme cleaves the last sugar unit of glycosphingolipids, including globotriaosylceramide $\left(\mathrm{Gb}_{3}\right)$, globotriaosylsphingosine (lyso- $\left.\mathrm{Gb}_{3}\right)$, and galabiosylceramide $\left(\mathrm{Ga}_{2}\right)$. Enzyme impairment leads to substrate accumulation in different organs, vascular endothelia, and biological fluids. Enzyme replacement therapy (ERT) is a commonly used treatment. Urinary analysis of $\mathrm{Gb}_{3}$ isoforms (different fatty acid moieties), as well as lyso-Gb and its analogues, is a reliable way to monitor treatment. These analogues correspond to lyso- $\mathrm{Gb}_{3}$ with chemical modifications on the sphingosine moiety $\left(-\mathrm{C}_{2} \mathrm{H}_{4},-\mathrm{C}_{2} \mathrm{H}_{4}+\mathrm{O},-\mathrm{H}_{2},-\mathrm{H}_{2}+\mathrm{O},+\mathrm{O},+\mathrm{H}_{2} \mathrm{O}_{2}\right.$, and $+\mathrm{H}_{2} \mathrm{O}_{3}$ ). The effects of sample collection time on urinary biomarker levels between ERT cycles were not previously documented. The main objective of this project was to analyze the aforementioned biomarkers in urine samples from seven Fabry disease patients (three treated males, three treated females, and one ERT-naïve male) collected twice a day (morning and evening) for 42 days (three ERT cycles). Except for one participant, our results show that the biomarker levels were generally more elevated in the evening. However, there was less variability in samples collected in the morning. No cyclic variations in biomarker levels were observed between ERT infusions.
\end{abstract}

Keywords: Fabry disease; diurnal variation; globotriaosylceramide; globotriaosylsphingosine; mass spectrometry; glycosphingolipids

\section{Introduction}

Fabry disease (FD) (OMIM no. 301500) is an X-linked monogenic lysosomal storage disorder. While males are often more affected than females, some females can be as severely affected as males due to X-chromosome inactivation [1]. The main clinical features of FD are cardiac hypertrophy, 
progressive renal deficiency, and increased risk of ischemic strokes. Other signs and symptoms include acroparesthesia, hypohidrosis, gastrointestinal involvement, angiokeratomas, cornea verticillata [2], and vascular tortuosities of the upper eyelid [3]. FD is caused by mutations in the GLA gene encoding the $\alpha$-galactosidase A enzyme (EC 3.2.1.22). The function of this glycoside hydrolase is to cleave the terminal $\alpha$-galactosyl moieties from glycoproteins or glycolipids [1]. The deficiency of the enzyme leads to the accumulation of substrates such as globotriaosylsphingosine (lyso-Gb $\mathrm{G}_{3}$ ), galabiosylceramide $\left(\mathrm{Ga}_{2}\right)$, and globotriaosylceramide $\left(\mathrm{Gb}_{3}\right)$ in cells [4], tissues [5], and biological fluids [6-9]. Different untargeted metabolomic studies performed in our laboratory with urine and plasma specimens from FD patients identified different analogues of lyso- $\mathrm{Gb}_{3}[10,11]$, as well as analogues/isoforms of $\mathrm{Ga}_{2}$ [12] and $\mathrm{Gb}_{3}$ [13], as FD biomarkers. The analogues correspond to molecules with modified sphingosine moieties, whereas the isoforms correspond to molecules with different fatty acid chains. As for lyso-Gb in urine, the following modifications on the sphingosine moiety were detected: $-\mathrm{C}_{2} \mathrm{H}_{4}(-28 \mathrm{Da})$, $-\mathrm{C}_{2} \mathrm{H}_{4}+\mathrm{O}(-12 \mathrm{Da}),-\mathrm{H}_{2}(-2 \mathrm{Da}),-\mathrm{H}_{2}+\mathrm{O}(+14 \mathrm{Da}),+\mathrm{O}(+16 \mathrm{Da}),+\mathrm{H}_{2} \mathrm{O}_{2}(+34 \mathrm{Da})$, and $+\mathrm{H}_{2} \mathrm{O}_{3}$ $(+50 \mathrm{Da})[10]$. In fact, most of the lyso-Gb 3 analogues in urine are more abundant than lyso-Gb itself. Previous results showed that FD children with the late-onset cardiac variant $p$.N215S mutation presented normal urinary levels of lyso-Gb $\mathrm{G}_{3}$, but abnormal levels of some lyso- $\mathrm{Gb}_{3}$ analogues [14]. Similarly, five patients with the $p . \mathrm{N} 215 \mathrm{~S}$ mutation presented abnormal levels of methylated $\mathrm{Gb}_{3}$ isoforms, but normal levels of non-methylated $\mathrm{Gb}_{3}$ isoforms [7]. Moreover, the analysis of urine samples from FD patients with the late-onset IVS4+919G > A cardiac variant mutation prevalent in Taiwan revealed that some lyso- $\mathrm{Gb}_{3}$ analogue levels had a positive association with the left-ventricular mass index and/or the Mainz Severity Score Index [15]. Chaperone therapy with migalastat (Galafold, Amicus Therapeutics) is now available for patients with amenable mutations [16]. However, the most common treatment for FD remains ERT [17], which consists of bi-weekly intravenous infusions of recombinant $\alpha$-galactosidase A such as agalsidase-alpha at $0.2 \mathrm{mg} / \mathrm{kg}$ (Replagal, Shire/Takeda Pharmaceutical) or agalsidase-beta at either 1.0 or $0.3 \mathrm{mg} / \mathrm{kg}$ (Fabrazyme, Sanofi Genzyme). A gene therapy clinical trial for FD is also ongoing [18]. The analysis of biomarkers in urine is a method of choice for monitoring the response of FD patients to ERT since sample collection is non-invasive. However, to the best of our knowledge, there are no published studies investigating the diurnal variation of urinary biomarkers and their fluctuation between ERT infusions. The main objectives of this research project were, thus, to evaluate if the urinary FD biomarker concentrations show statistically significant differences when sampled in the morning compared to the evening, and if the levels fluctuate periodically between ERT infusions using bi-daily monitoring over three ERT cycles (42 days).

\section{Results}

\subsection{Biomarker Measures according to the Collection Time}

\subsubsection{Mean Measured Biomarker Levels}

Raw data are available in Supplemental Table S1. FD biomarkers are expressed as mean measured levels for each collection time point (Figure 1). Paired-sample $t$-tests were used to determine whether there was a statistically significant mean difference between the biomarker concentrations measured in specimens collected in the morning compared to specimens collected in the evening. Results are presented in Table 1 for each participant, along with a mean \pm one standard deviation for each collection time. Results show that the measured concentrations might be influenced by the collection time for some biomarkers. Our results show a statistically significant mean difference for lyso-Gb $\mathrm{Gb}_{3}$ levels in four participants $(1,2,6$, and 7$)$, where concentrations were higher in urine specimens collected in the evening compared to those collected in the morning. Similar results were obtained for lyso- $\mathrm{Gb}_{3}$ analogue ( $-2 \mathrm{Da}$ ) in participants $1,2,5$, and 6 , and for $\mathrm{Gb}_{3}$ in participants 2 and 5 . Regarding lyso-Gb analogues $(-12 \mathrm{Da}),(+14 \mathrm{Da}),(+16 \mathrm{Da}),(+34 \mathrm{Da})$, and $(+50 \mathrm{Da})$, there were no mean differences in the measured levels based on the collection time, except for patient 3 , where the measured values 
had a tendency to be higher in morning specimens. Finally, there were mixed results for the lyso- $\mathrm{Gb}_{3}$ analogue $(-28 \mathrm{Da})$, where the measured values were higher in the evening for participants 2 and 5 , but higher in the morning for participant 4.

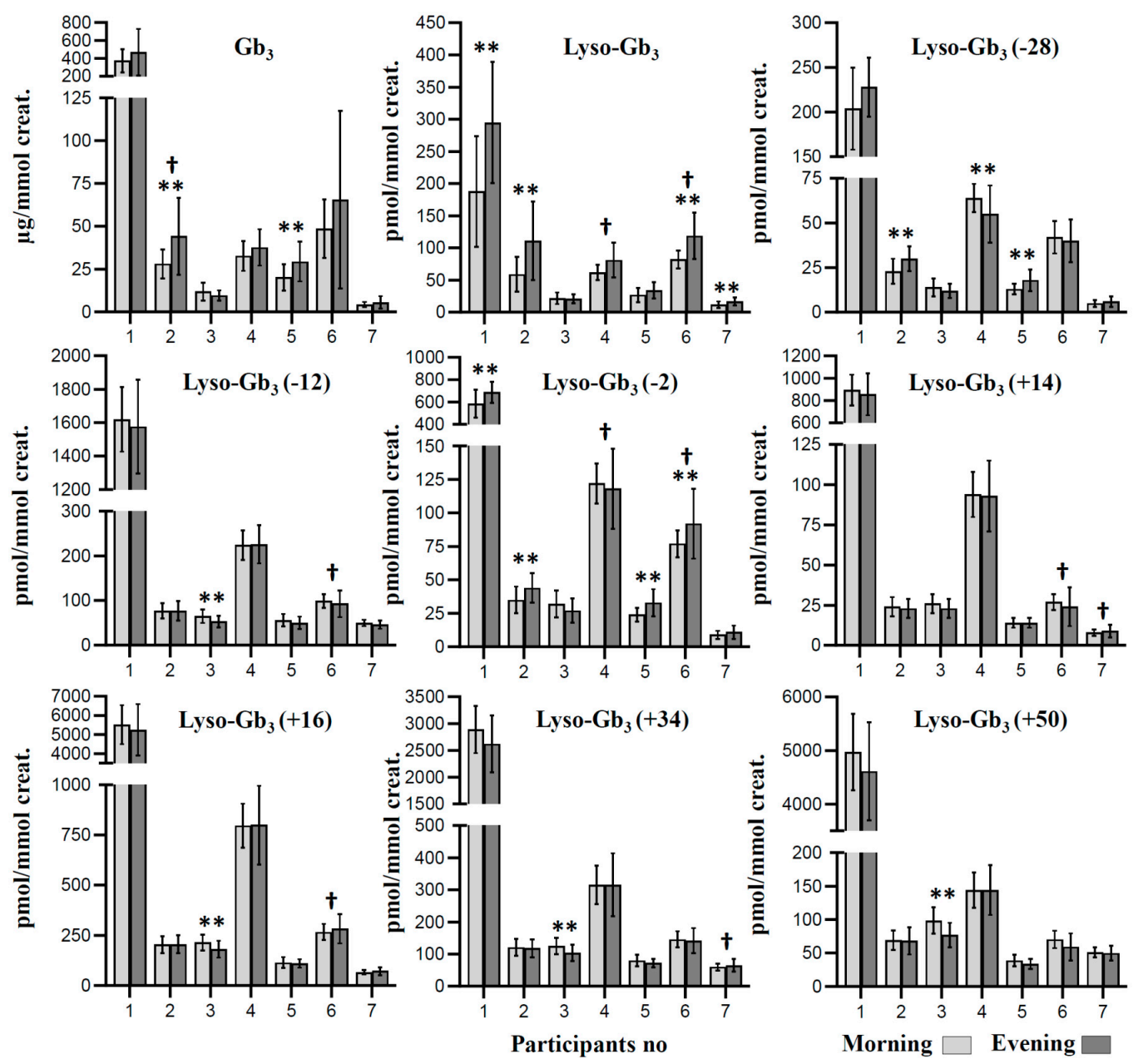

Figure 1. Mean concentration of Fabry biomarkers for three enzyme replacement therapy (ERT) cycles ( $n=42$ days) at two different collection times in seven study participants. All participants were under ERT, except participant no. 1 who never received treatment. Patients $1-4$ were males, whereas patients 5-7 were females. For lyso-Gb 3 analogues, the mass difference in Da compared to lyso-Gb 3 is indicated in brackets. ${ }^{* *}$ corrected $p$-value $\leq 0.001$ for the paired-sample $t$-test; + corrected $p$-value $\leq 0.001$ for Levene's test for equality of variances.

\subsubsection{Variance of Biomarker Measures}

Levene's test for equality of variances was used to verify the homogeneous variance of biomarker levels when urine specimens were collected either in the morning or in the evening. Results are shown in Table 2 for each collection time, along with relative standard deviations (RSDs) ( $n=42$ days). In general, RSDs were higher for specimens collected in the evening. The variance was equivalent for the morning and evening specimens for each biomarker according to Levene's test for participants 1,3 , and 5 . However, $\mathrm{Gb}_{3}$ levels had more variance in evening specimens for participant 2, while lyso- $\mathrm{Gb}_{3}$ and the analogue ( $-2 \mathrm{Da}$ ) levels had more variance in evening specimens for participant 4 . For participant 6, our results show that five biomarkers (lyso-Gb ${ }_{3}$, and analogues $(-12 \mathrm{Da}),(-2 \mathrm{Da})$, $(+14 \mathrm{Da})$, and $(+16 \mathrm{Da}))$ had a higher variance for specimens collected in the evening. The same tendency was observed for lyso-Gb 3 analogues (+14 Da) and (+34 Da) for participant 7. 
Table 1. Paired-sample $t$-tests assessing mean differences in measured biomarker levels over 42 days in seven Fabry patients at two different collection time points (morning and evening). Means \pm standard deviation are shown. For statistically significant results, $p$-values are highlighted in gray or in black if the highest mean concentration value was measured in the morning or the evening, respectively.

\begin{tabular}{|c|c|c|c|c|c|c|c|c|c|c|c|c|c|c|c|c|c|c|c|c|c|}
\hline & \multicolumn{3}{|c|}{ Participant 1} & \multicolumn{3}{|c|}{ Participant 2} & \multicolumn{3}{|c|}{ Participant 3} & \multicolumn{3}{|c|}{ Participant 4} & \multicolumn{3}{|c|}{ Participant 5} & \multicolumn{3}{|c|}{ Participant 6} & \multicolumn{3}{|c|}{ Participant 7} \\
\hline & M & $\mathrm{E}$ & $p$ & M & E & $p$ & $\mathrm{M}$ & $\mathrm{E}$ & $p$ & M & $\mathrm{E}$ & $p$ & M & $\mathrm{E}$ & $\mathrm{p}$ & M & $\mathrm{E}$ & $\mathrm{p}$ & M & $\mathrm{E}$ & $\mathrm{p}$ \\
\hline $\mathrm{Gb}_{3}$ & $373 \pm 128$ & $468 \pm 261$ & $<0.050$ & $28 \pm 8$ & $44 \pm 23$ & $<0.001^{\dagger}$ & $12 \pm 5$ & $10 \pm 3$ & $<0.050$ & $33 \pm 9$ & $38 \pm 11$ & $<0.050$ & $20 \pm 8$ & $29 \pm 12$ & $<0.001$ & $49 \pm 17$ & $66 \pm 52$ & 0.059 & $4 \pm 2$ & $6 \pm 4$ & 0.060 \\
\hline & $188 \pm 86$ & $295 \pm 94$ & $<0.001^{+}$ & $59 \pm 27$ & $111 \pm 61$ & $<0.001^{\dagger}$ & $22 \pm 9$ & $21 \pm 7$ & 0.525 & $62 \pm 12$ & $81 \pm 27$ & $<0.050$ & $27 \pm 11$ & $34 \pm 13$ & $<0.050$ & $82 \pm 14$ & $119 \pm 36$ & $<0.001^{\top}$ & $12 \pm 5$ & $17 \pm 6$ & $<0.001^{+}$ \\
\hline Lyso-Gb $(-28)$ & $204 \pm 46$ & $228 \pm 33$ & $<0.050$ & $23 \pm 7$ & $30 \pm 7$ & $<0.001^{\dagger}$ & $14 \pm 5$ & $12 \pm 4$ & $<0.050$ & $64 \pm 8$ & $55 \pm 16$ & $0.001^{\dagger}$ & $13 \pm 3$ & $18 \pm 6$ & $<0.001^{\dagger}$ & $42 \pm 9$ & $40 \pm 12$ & 0.224 & $5 \pm 2$ & $6 \pm 3$ & 0.208 \\
\hline Lyso-Gb $3(-12)$ & $1620 \pm 193$ & $1576 \pm 281$ & 0.282 & $77 \pm 17$ & $77 \pm 22$ & 0.894 & $65 \pm 15$ & $53 \pm 13$ & $<0.001^{\dagger}$ & $224 \pm 33$ & $226 \pm 43$ & 0.831 & $56 \pm 14$ & $50 \pm 15$ & $<0.050$ & $99 \pm 15$ & $93 \pm 30$ & 0.224 & $50 \pm 7$ & $46 \pm 9$ & $<0.050$ \\
\hline Lyso-Gb $3(-2)$ & $585 \pm 126$ & $689 \pm 95$ & $<0.001^{\dagger}$ & $35 \pm 10$ & $44 \pm 11$ & $<0.001+$ & $32 \pm 10$ & $27 \pm 9$ & $<0.050$ & & & 0.39 & $24 \pm 5$ & $33 \pm 10$ & $<0.001^{\dagger}$ & $77 \pm 10$ & & $0.001^{\dagger}$ & & $11 \pm 5$ & 0.135 \\
\hline Lyso-Gb $(+14)$ & $894 \pm 138$ & $855 \pm 188$ & $<0.050$ & $24 \pm 6$ & $23 \pm$ & 0.105 & $26=$ & $23=$ & $<0.0$ & $94 \pm$ & & 0.4 & & & & $27 \pm 5$ & & 0.280 & $8 \pm 2$ & & 0.457 \\
\hline Lyso-Gb $3(+16)$ & $5524 \pm 1017$ & $5246 \pm 1351$ & $<0.050$ & $204 \pm 43$ & $206 \pm 45$ & 0.760 & $215 \pm 39$ & $182 \pm 42$ & $<0.001^{\dagger}$ & $797 \pm 109$ & $800 \pm 196$ & 0.927 & $115 \pm 27$ & $110 \pm 21$ & 0.2 & $267 \pm 40$ & $283 \pm 73$ & 0.234 & $67 \pm 11$ & $72 \pm 20$ & 0.094 \\
\hline Lyso-Gb $3(+34)$ & $2892 \pm 440$ & $2622 \pm 532$ & 0.001 & $121 \pm 26$ & $118 \pm 28$ & 0.527 & $125 \pm 25$ & $104 \pm 26$ & $<0.001^{\dagger}$ & $315 \pm 60$ & & 0.797 & $80 \pm 18$ & $72 \pm$ & $<0.050$ & $146 \pm 25$ & $142 \pm 39$ & 0.609 & $60 \pm 11$ & $65 \pm 20$ & 0.137 \\
\hline Lyso-Gb $3(+50)$ & $4973 \pm 711$ & $4613 \pm 913$ & $<0.050$ & $69 \pm 15$ & $68 \pm 20$ & 0.779 & $99 \pm 20$ & $77 \pm 18$ & $<0.001^{\dagger}$ & $144 \pm 27$ & $144 \pm 37$ & 0.847 & $39 \pm 9$ & $34 \pm 8$ & $<0.050$ & $70 \pm 13$ & $59 \pm 20$ & $<0.050$ & $51 \pm 7$ & $50 \pm 11$ & 0.583 \\
\hline
\end{tabular}

${ }^{+}$indicates that statistical significance was still valid after correction for multiple comparisons using the Holm-Š́íák procedure (63 comparisons); M: morning collection; E: evening collection. All participants were under ERT except for participant no. 1 who was an ERT-naïve patient. Patients $1-4$ were males, whereas patients $5-7$ were females. For lyso-Gb 3 analogues, the mass difference in Da compared to lyso- $\mathrm{Gb}_{3}$ is indicated in brackets. 


\subsection{Longitudinal Follow-Up}

\subsubsection{Untreated Fabry Disease Participant}

The longitudinal biomarker follow-up of participant no. 1 was useful to evaluate the variability of the measured biomarker concentrations in urine specimens from an untreated Fabry patient. Longitudinal follow-ups are shown in Figure 2. According to RSDs, the dispersion of the measured concentrations $(n=42)$ was higher for lyso-Gb 3 (morning RSD: $46 \%$; evening RSD: $32 \%$ ) and $\mathrm{Gb}_{3}$ (morning RSD: $34 \%$; evening RSD: $56 \%$ ) compared to lyso-Gb $\mathrm{Gb}_{3}$ analogues with RSDs $\leq 23 \%$ in urine specimens collected in the morning, and RSDs $\leq 26 \%$ in urine specimens collected in the evening.

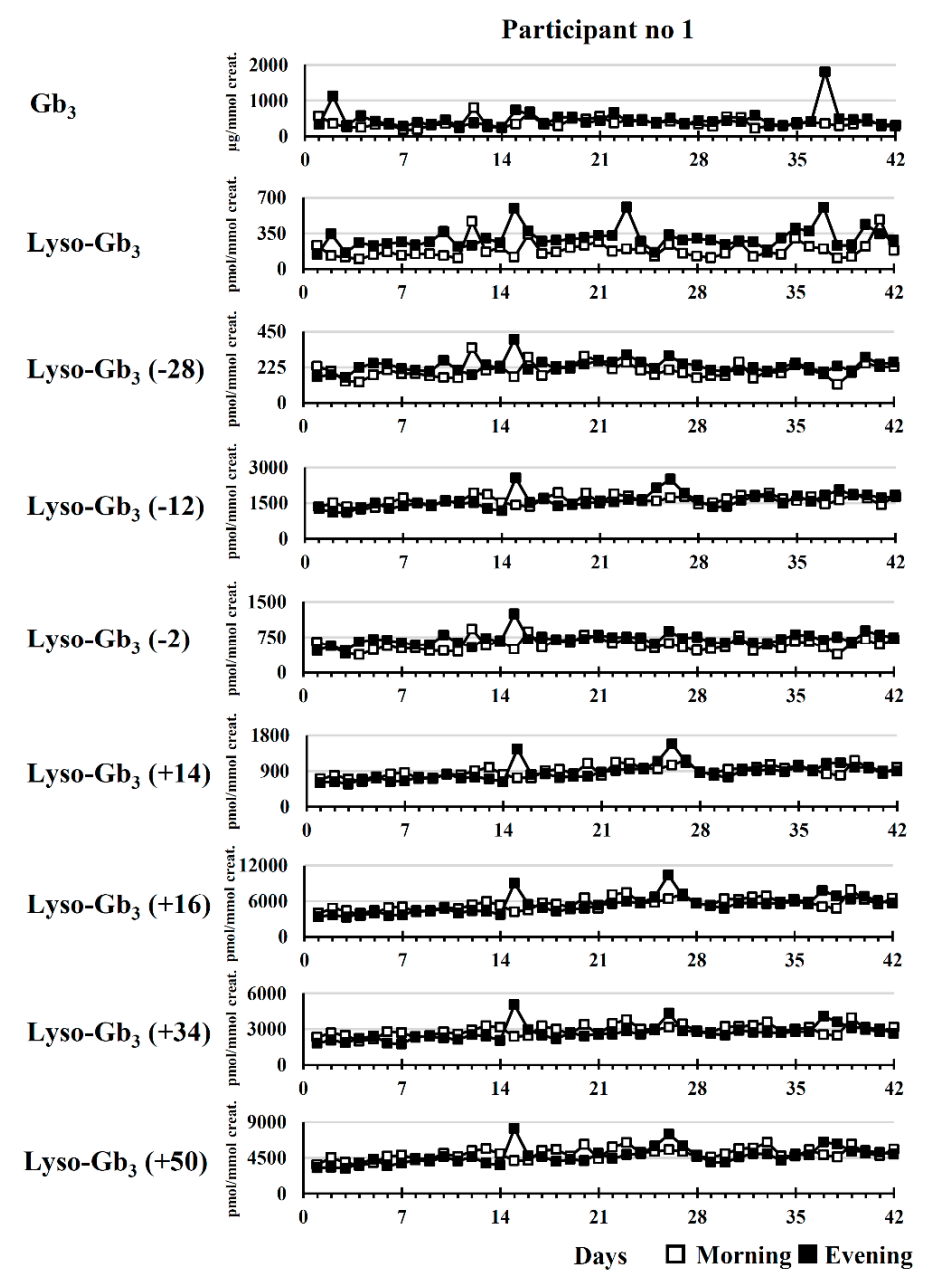

Figure 2. Longitudinal variation $(n=42)$ of Fabry biomarker levels in a 30-year-old untreated male (no ERT) (participant no. 1). For lyso-Gb 3 analogues, the mass difference in Da compared to lyso-Gb is indicated in brackets.

\subsubsection{Participants Treated with ERT}

Considering that biomarker levels had equivalent or lower variance in urine specimens collected in the morning compared to urine specimens collected in the evening, results of longitudinal biomarker follow-ups in treated males and females are shown in specimens collected in the morning only. 
Table 2. Levene's test for equality of variances examining the influence of urine collection time on the variability of the measured biomarker levels over 42 days in seven Fabry patients. Relative standard deviations (RSDs) are shown $(n=42)$ for each collection time. For statistically significant results, $p$-values are highlighted in gray or in black if the highest variance was obtained in the morning or in the evening, respectively.

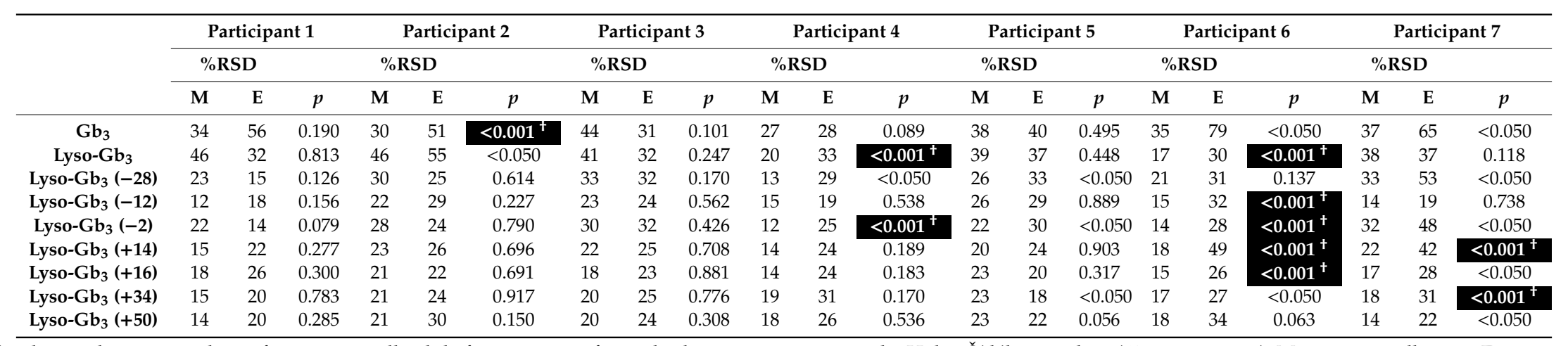

${ }^{\dagger}$ indicates that statistical significance was still valid after correction for multiple comparisons using the Holm-Š́́dák procedure (63 comparisons); M: morning collection; E: evening collection; RSD: relative standard deviation. For lyso-Gb $\mathrm{G}_{3}$ analogues, the mass difference in Da compared to lyso-Gb 3 is indicated in brackets. 
Male Fabry Disease Participants

Longitudinal biomarker follow-ups are shown in Figure 3 for ERT-treated male participants 2, 3, and 4 .

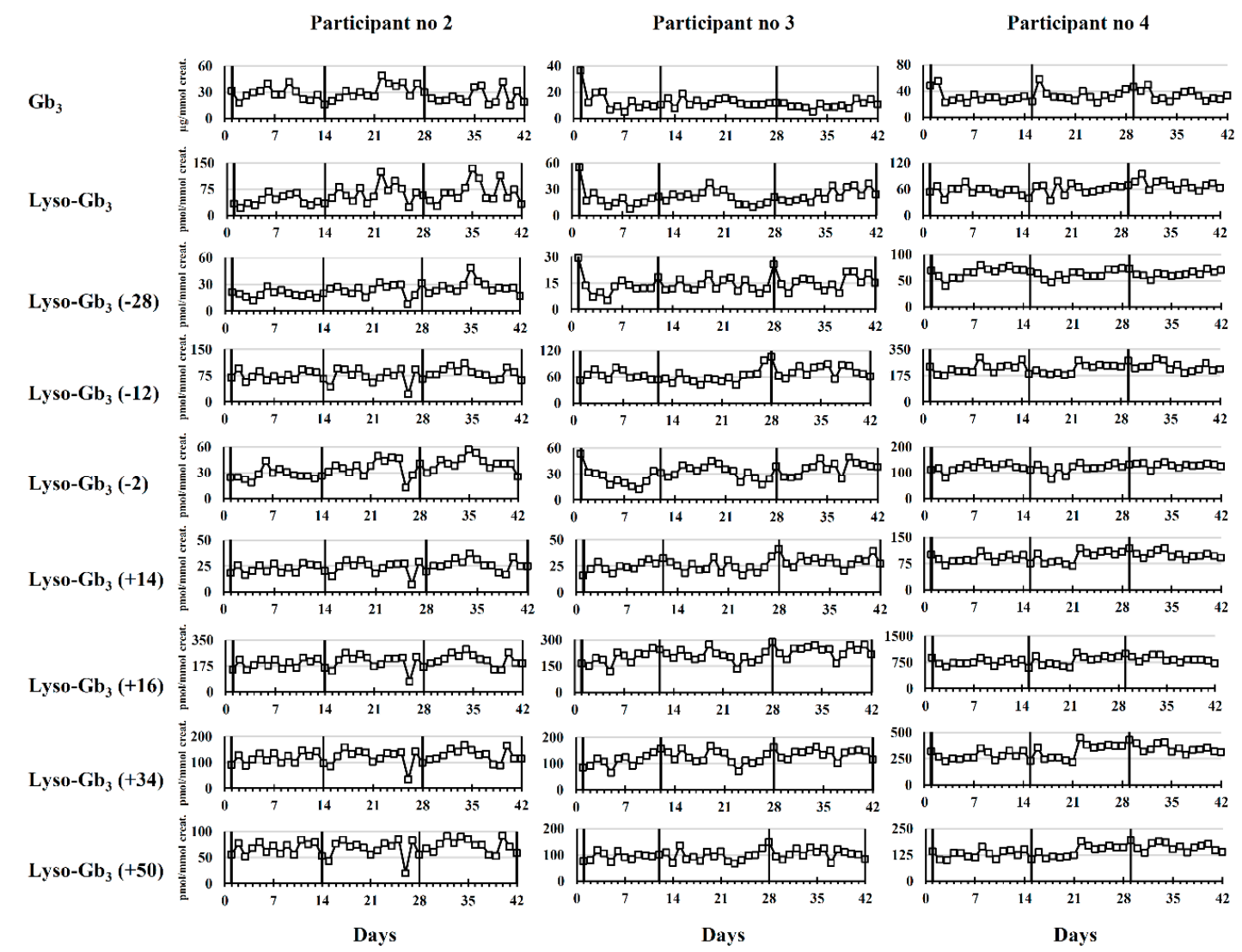

Figure 3. Longitudinal variation $(n=42)$ of Fabry disease biomarker levels during three ERT cycles in male patients. Results are shown for morning urine collection only. For lyso- $\mathrm{Gb}_{3}$ analogues, the mass difference in Da compared to lyso- $\mathrm{Gb}_{3}$ is indicated in brackets. Vertical lines indicate the time points where Fabry patients received their ERT infusions.

Visual inspection of longitudinal follow-ups in Figure 3 suggests that, in general, the biomarker concentrations were stable in male patients during the study and oscillated around a mean biomarker value. In fact, no clear pattern of cyclic variation was observed in relation to ERT infusions. A visual examination of longitudinal follow-ups also showed that lyso-Gb3 analogues (-12 Da), (+14 Da), (+16 Da), (+34 Da), and (+50 Da) had similar profiles.

Female Fabry Disease Participants

Longitudinal biomarker follow-ups are shown in Figure 4 for female participants 5, 6, and 7 who were all ERT-treated.

Our results show that there was no clear pattern of cyclic biomarker variation observed in urine specimens of female participants during three cycles of ERT infusions. 


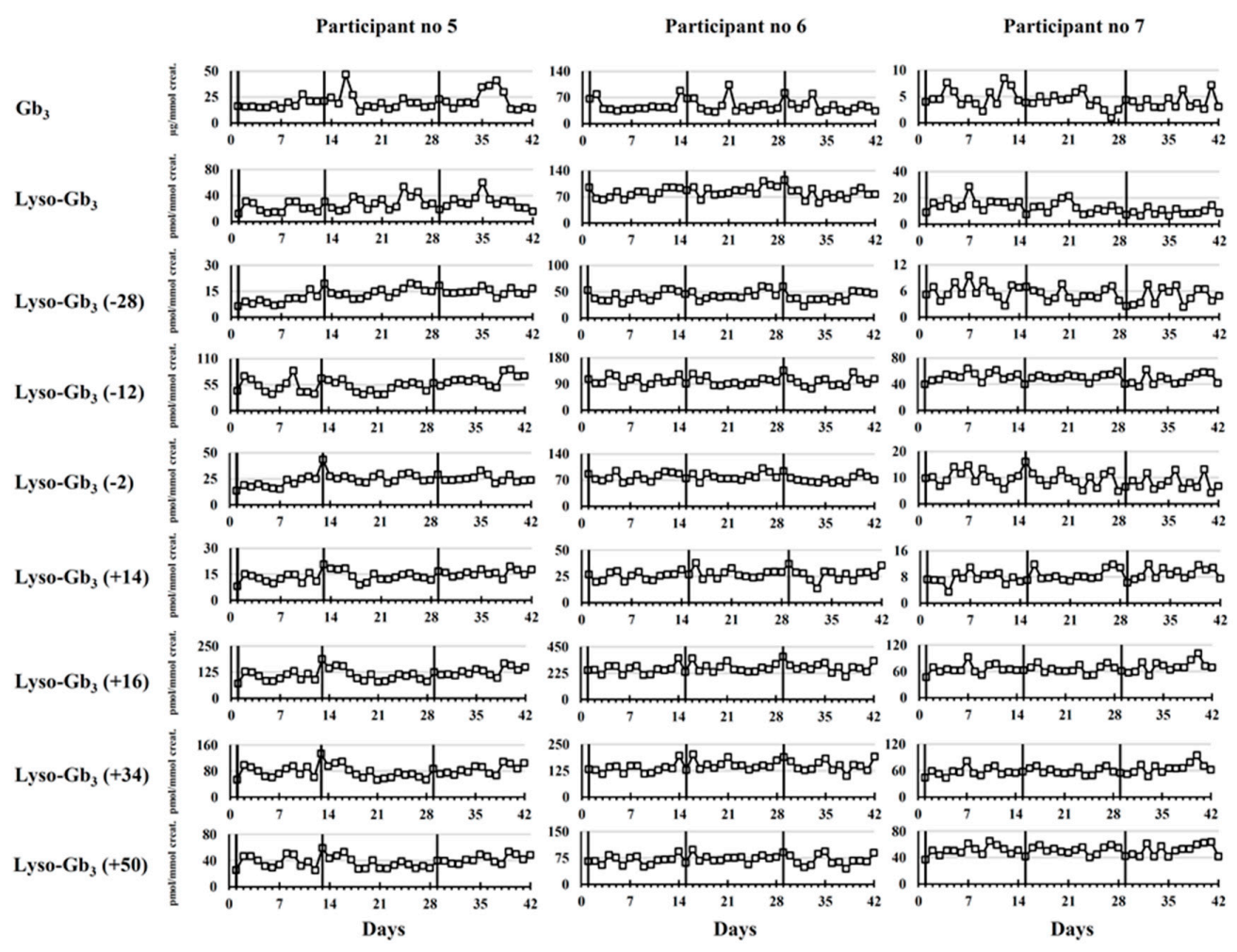

Figure 4. Longitudinal variation $(n=42)$ of Fabry disease biomarker levels during three ERT cycles in female patients. Results are shown for morning urine collection only. The vertical lines indicate the time points where Fabry patients received their ERT infusions. For lyso-Gb 3 analogues, the mass difference in Da compared to lyso- $\mathrm{Gb}_{3}$ is indicated in brackets.

\section{Discussion}

The aim of the present study was to evaluate the variation of FD biomarkers, such as $\mathrm{Gb}_{3}$, lyso- $\mathrm{Gb}_{3}$, and related analogues, in urine specimens collected by seven participants diagnosed with FD (one untreated and six ERT-treated). Urine specimens were collected daily, at two different timepoints, for a duration of 42 days. The collection time (morning or evening urine specimens) and the administration of ERT infusions were evaluated as potential variability factors. A total of 84 urine specimens were collected at home by each study participant. Patient compliance was excellent as no urine specimen collection was missed. Two shipments with thawed urine specimens were received from participant no. 3 (day 26 to day 42). However, this was not an issue considering that $\mathrm{Gb}_{3}$ and lyso- $\mathrm{Gb}_{3}$ and related analogues are stable for at least $24 \mathrm{~h}$ at room temperature in urine specimens $[7,9]$. Urine specimens offer many advantages for biomarker analyses. Collection is safe and can be performed in a non-invasive way by patients, without the need for trained personnel such as phlebotomists. The analysis of spot urine specimens is more convenient compared to 24-h urine collection specimens, but results can vary according to the rate of urine production. Creatinine, a compound excreted at a constant rate in urine through glomerular filtration [19], is commonly used to normalize results, which are then compared with age- and gender-matched reference values. $\mathrm{Gb}_{3}$ normalization with creatinine might be arguable, considering that its presence in urine does not originate from glomerular filtration, but from the shedding of renal tubular cells and podocytes in urine [20]. Consequently, $\mathrm{Gb}_{3}$ normalization with membrane lipids such as sphingomyelin or phosphatidylcholine was previously investigated. Mills and colleagues [21] showed that the distribution of $\mathrm{Gb}_{3}$-to-sphingomyelin ratios was similar to the distribution of the $\mathrm{Gb}_{3}$-to-creatinine ratios. Whitfield and colleagues had the same conclusions regarding phosphatidylcholine [22]. Nevertheless, precautions should always be taken for the analysis of $\mathrm{Gb}_{3}$ in urine since urine specimens should be homogenized carefully, and filter papers 
impregnated with urine should be saturated and dried horizontally to avoid unequal distribution of $\mathrm{Gb}_{3}$ and creatinine molecules.

Results obtained following the paired-sample $t$-tests show that the collection time might influence the biomarker levels in some cases. The measured values were generally more elevated in specimens collected in the evening, except for participant no. 3. Interestingly, there was no difference in lyso-Gb analogue (+14 Da) urinary levels depending on the collection time in all participants. This might indicate that this specific biomarker could be useful for long-term follow-up when it is not possible to collect urine specimens at a specific time. The results obtained following Levene's test for equality of variances revealed that there was usually more biomarker level variability in the evening urine specimens. Specimens collected in the evening might be more prone to variations due to external factors such as physical activity, food intake, and water consumption. The results also showed that the concentrations of urinary lyso- $\mathrm{Gb}_{3}$ and $\mathrm{Gb}_{3}$ measured longitudinally were subject to more variability in the untreated patient than the measured concentrations of lyso-Gb $b_{3}$ analogues, according to RSDs ( $n=42$ days). A similar trend was observed in treated patients. This might indicate that the urinary levels of lyso- $\mathrm{Gb}_{3}$ analogues are more reliable and less variable for long-term follow-up and monitoring of FD patients than $\mathrm{Gb}_{3}$ and lyso-Gb $\mathrm{G}_{3}$ itself. Regarding the variability of biomarker concentrations between ERT infusions, we initially hypothesized a cyclic biomarker variation with reduced biomarker levels immediately after infusion, followed by a slow increase over the rest of the 14-day cycle. A visual examination of Figures 3 and 4 does not corroborate this initial hypothesis. Considering these results, urine specimens collected in the morning should be preferred over urine specimens collected in the evening for the quantification of FD biomarkers as part of a longitudinal evaluation. The results obtained do not suggest that urine specimens need to be collected at a specific time between two ERT infusions, since this was not a factor of variability in the present study. Urinary lyso-Gb $\mathrm{G}_{3}$ analogues might be more reliable for longitudinal follow-up compared to lyso- $\mathrm{Gb}_{3}$ and $\mathrm{Gb}_{3}$, considering that some of these analogues showed less variability depending on the collection time than lyso- $\mathrm{Gb}_{3}$ or $\mathrm{Gb}_{3}$. It is noteworthy to mention that the analysis of lyso- $\mathrm{Gb}_{3}$ analogues is particularly relevant in urine, considering that lyso-Gb $\mathrm{G}_{3}$ represents only approximately $2 \%$ of all lyso-Gb $b_{3}$-related species in this matrix. In comparison, lyso-Gb $b_{3}$ represents approximately $57 \%$ of all lyso-Gb $b_{3}$-related species in plasma $[8,9]$.

\section{Conclusions}

According to the results obtained in this study, there is no cyclic variation of the urinary Fabry disease biomarkers (Lyso- $\mathrm{Gb}_{3}$ and analogues, and $\mathrm{Gb}_{3}$ ) normalized to creatinine between two ERT cycles. The diurnal variation observed for the urinary Fabry disease biomarkers normalized to creatinine might be attributed not only to the sphingolipid levels, but also to the variation in the creatinine levels. Nevertheless, the biomarker/creatinine ratios show less variation in urine specimens collected in the morning. Moreover, the lyso- $\mathrm{Gb}_{3}$ analogue/creatinine ratios show less variation than the lyso- $\mathrm{Gb}_{3} /$ creatinine and $\mathrm{Gb}_{3} /$ creatinine ratios. Finally, this study suggests that the urinary Fabry disease biomarker/creatinine ratios are more elevated in specimens collected in the evening except for the lyso- $\mathrm{Gb}_{3}$ analogue (+14 Da) which shows similar abundance at both collection times.

\section{Materials and Methods}

\subsection{Ethics Approval}

This multicenter research project was approved by the Research Ethics Board (REB) at the Faculty of Medicine and Health Sciences at the Centre Intégré Universitaire de Santé et de Services Sociaux de l'Estrie-Centre Hospitalier Universitaire de Sherbrooke (CIUSSSE-CHUS) (REB no 06-011, Approval date: 22 February 2006) and at the CIUSSS du Nord-de-l'Île-de-Montréal-Hôpital du Sacré-Coeur de Montréal (REB no 2014-1071, Approval date: 16 October 2014). All subjects provided informed consent for inclusion before their participation in the study. The study was conducted in accordance with the Declaration of Helsinki. 


\subsection{Recruitment of Participants}

Seven FD patients who were confirmed either by demonstrating a marked enzyme deficiency of $\alpha$-galactosidase A and/or via GLA gene mutation analysis. They were recruited from two centers in the province of Quebec: CIUSSSE-CHUS (Sherbrooke, QC, Canada) and CIUSSS du Nord-de-l'Île-de-Montréal-Hôpital du Sacré-Coeur de Montréal (Montreal, QC, Canada). Participant demographics are shown in Table 3. All participants, except one, were receiving ERT.

\subsection{Urine Specimen Collection}

Urine specimen collection was performed at home by participants. Each participant received the following urine collection kit: 86 conical centrifuge tubes in polypropylene $(50 \mathrm{~mL})$ divided into four Styrofoam racks, four pre-addressed shipping boxes, eight icepacks, parafilm, and four large plastic bags. Two urine specimens (identified as $\mathrm{M}$ and $\mathrm{E}$ ) were collected each day for a duration of 42 days, representing three ERT cycles for treated patients. Specimen M was collected in the morning (before breakfast), and specimen E was collected in the evening, after dinner. The collection of the first urine specimen was performed when ERT-treated patients received their day- 1 infusion. Parafilm was used to seal the cap of the tube after urine collection, and the tube was frozen immediately. Collected urine specimens $(n=25)$ were placed in a Styrofoam rack and inserted in a large plastic bag in a pre-addressed box containing two icepacks. Samples were picked up by a shipping courier and delivered to our laboratory in Sherbrooke, QC for analysis.

\subsection{Analysis of Fabry Disease Biomarkers}

FD biomarkers were analyzed as mentioned below using assays previously devised and validated in our laboratory. Intra-assay and inter-assay precisions were good with relative standard deviations (RSDs) $\leq 15 \%$.

\subsection{1. $\mathrm{Gb}_{3}$}

Total $\mathrm{Gb}_{3}$ was measured (total ion count (TIC)) for C16:0, C18:0, C20:0, C22:1, C22:0, C24:1, C24:0, and C24:OH isoforms simultaneously with creatinine in dried urine spots (DUS) as previously described [23,24]. Briefly, urine specimens were mixed carefully; then, $1 \mathrm{~mL}$ was deposited on a 5-cm-diameter disc of Whatman-GE 903 filter paper and dried completely at room temperature for at least $4 \mathrm{~h}$. DUS extraction was performed by adding $4 \mathrm{~mL}$ of methanol to the filter paper disc and shaking for $1 \mathrm{~h}$ with an orbital shaker (300 rpm). Next, $10 \mu \mathrm{L}$ of the extract was analyzed by high-performance liquid chromatography (HPLC) coupled to tandem mass spectrometry (MS/MS). The normal reference value was defined as $\leq 25 \mu \mathrm{g} / \mathrm{mmol}$ creatinine. Urine sample aliquots from an untreated Fabry male and an untreated Fabry female were used as high- and low-quality controls for this study. The intra- and interday precision values measured (RSD\%) were respectively $\leq 12.5 \%$ and $\leq 11.3 \%$ for $\mathrm{Gb}_{3}$ and $\leq 10.1 \%$ and $\leq 7.0 \%$ for creatinine [24]. The intra- and interday accuracy values (Bias\%) measured for $\mathrm{Gb}_{3}$ at a concentration of $3.75 \mu \mathrm{g} / \mathrm{mL}$ were respectively $4.3 \%$ and $7.9 \%$.

\subsection{2. $\mathrm{Lyso}-\mathrm{Gb}_{3}$ and Related Analogues}

Lyso-Gb $b_{3}$ and seven related analogues were analyzed by ultra-performance liquid chromatography (UPLC) coupled to MS/MS, following solid-phase extraction with Oasis mixed-mode strong cation exchange (MCX) cartridges (Waters Corp., Milford, MA, USA), as previously described $[9,25]$. Normal reference values were $0 \mathrm{pmol} / \mathrm{mmol}$ creatinine (not detected) for lyso- $\mathrm{Gb}_{3}$ and the analogues $(-28 \mathrm{Da})$, $(-12 \mathrm{Da}),(-2 \mathrm{Da})$, and (+14 Da). Normal reference values were 17, 14, and $55 \mathrm{pmol} / \mathrm{mmol}$ creatinine for the lyso-Gb $\mathrm{Gb}_{3}$ analogues (+16 Da), (+34 Da), and (+50 Da), respectively. Urine sample aliquots from an untreated Fabry male and an untreated Fabry female were used as high- and low-quality controls for this study. For lyso-Gb 3 and its seven analogues, the intra- and interday assay precision values (RSD\%) were respectively $\leq 13.0 \%$ and $\leq 20.1 \%$. The intraday and interday accuracy values (Bias $\%$ ) for lyso-Gb $b_{3}$ at concentrations of $1500,11,000$, and $24,000 \mathrm{pM}$ were $<7.9 \%$ [9]. 
Table 3. Participant demographics. n/a: not applicable; ERT: enzyme replacement therapy; eGFR: estimated glomerular filtration rate; CKD-EPI: Chronic Kidney Disease Epidemiology Collaboration (CKD-EPI) equation.

\begin{tabular}{|c|c|c|c|c|c|c|c|}
\hline Patient No & 1 & 2 & 3 & 4 & 5 & 6 & 7 \\
\hline Gender & $\mathrm{M}$ & $\mathrm{M}$ & $\mathrm{M}$ & $\mathrm{M}$ & $\mathrm{F}$ & $\mathrm{F}$ & $\mathrm{F}$ \\
\hline Age (years) & 30 & 28 & 40 & 36 & 49 & 57 & 67 \\
\hline Treatment status & No treatment & Replagal & Fabrazyme & Fabrazyme & Replagal & Replagal & Fabrazyme \\
\hline Mutation & c.17_327del & c. $612 \mathrm{G}>\mathrm{A}$ & c.35_47del13 & c. $1241 \mathrm{~T}>\mathrm{C}$ & c. $1042 \mathrm{G}>\mathrm{C}$ & c. $1241 \mathrm{~T}>\mathrm{C}$ & c. $877 \mathrm{C}>\mathrm{A}$ \\
\hline ERT start date & $\mathrm{n} / \mathrm{a}$ & $2005-07-26$ & $2008-09-30$ & 2003-05-31 & 2009-01-14 & 2011-02-02 & 2003-04-15 \\
\hline Serum creatinine $(\mu \mathrm{mol} / \mathrm{L})$ & 97 & 78 & 97 & 96 & 57 & 76 & 92 \\
\hline eGFR (CKD-EPI) $\left(\mathrm{mL} / \mathrm{min} / 1.73 \mathrm{~m}^{2}\right)$ & 91 & 116 & 83 & 87 & 105 & 76 & 56 \\
\hline Urinary proteins $(\mathrm{g} / 24 \mathrm{~h})$ & 1.07 & 0.14 & 1.19 & 1.38 & 0.25 & 3.21 & 0.07 \\
\hline
\end{tabular}




\subsection{Statistical Analyses}

Statistical analyses were performed using IBM SPSS Statistics version 24, while bar charts were done using GraphPad Prism version 8.2.1. Paired-sample $t$-tests were performed to evaluate if the mean difference of the measured biomarker concentration was significantly different from zero depending on the collection time. Shapiro-Wilk's test and visual inspection of a normal quantile-quantile (Q-Q) plot were used to determine if the differences in biomarker levels between the two collection times were normally distributed. If not, a mathematical transformation was applied to obtain normally distributed data. Levene's test for equality of variances was performed to evaluate if the variance of the measured biomarker levels was equal for both collection times over 42 days.

For all analyses, statistical significance was established at $p \leq 0.05$, and the Holm-Šídák procedure was used for multiple comparisons [26]. Specimen E, day 15 from patient 1 was excluded from statistics for lyso- $\mathrm{Gb}_{3}+$ analogue results only, considering that the lyso- $\mathrm{Gb}_{3}$ internal standard (IS) was off $(-48 \%)$ for this specimen, leading to falsely elevated results. Specimen E, day 34 from patient 7 was also excluded from statistics, considering that it was an extreme outlier ( $>3$ interquartile ranges from the 75 th percentile). This might be related to a low creatinine value in this sample ( $1.8 \mathrm{mmol} / \mathrm{L})$, leading to unreliable results.

Supplementary Materials: The following are available online at http://www.mdpi.com/1422-0067/21/17/6114/s1, Table S1. Raw study dataset; ERT: enzyme replacement therapy.

Author Contributions: Conceptualization and design of the study, C.A.-B.; methodology, C.A.-B., P.L., M.B., M.-F.A., C.F., and C.M.; validation, C.A.-B., P.L., and M.B.; analysis of samples, M.A., A.T., M.E.-E., and I.M.; resources, C.A.-B., B.M., and D.G.B.; data curation, C.A.-B.; writing-original draft preparation, P.L., and M.B.; writing-review and editing, M.B., P.L., M.A., A.T., M.E.-E., I.M., M.-F.A., C.F., C.M., D.G.B., B.M., and C.A.-B.; supervision, C.A.-B.; project administration, C.A.-B., M.-F.A., C.F., C.M., and D.G.B.; funding acquisition, C.A.-B. All authors read and agreed to the published version of the manuscript.

Funding: This study was funded by personal research funds from C. Auray-Blais.

Acknowledgments: We would like to thank Waters Corporation for their continued technical and scientific support.

Conflicts of Interest: The authors declare no conflict of interest.

\section{Abbreviations}

CIUSSSE- Centre Intégré Universitaire de Santé et de Services Sociaux de l'Estrie-Centre Hospitalier

CHUS Universitaire de Sherbrooke

DUS Dried urine spots

ERT Enzyme replacement therapy

FD Fabry disease

$\mathrm{Ga}_{2} \quad$ Galabiosylceramide

$\mathrm{Gb}_{3} \quad$ Globotriaosylceramide

HPLC High-performance liquid chromatography

LC-MS/MS Liquid chromatography tandem mass spectrometry

Lyso-Gb $\mathrm{Gb}_{3} \quad$ Globotriaosylsphingosine

MCX Mixed-mode strong cation exchange

MS/MS Tandem mass spectrometry

REB Research Ethics Board

RSD Relative standard deviation

TIC Total ion count

UPLC Ultra-performance liquid chromatography

\section{References}

1. Juchniewicz, P.; Kloska, A.; Tylki-Szymanska, A.; Jakobkiewicz-Banecka, J.; Wegrzyn, G.; Moskot, M.; Gabig Ciminska, M.; Piotrowska, E. Female fabry disease patients and X-chromosome inactivation. Gene 2018, 641, 259-264. [CrossRef] [PubMed]

2. Clarke, J.T.R. Narrative review: Fabry disease. Ann. Intern. Med. 2007, 146, 425-433. [CrossRef] 
3. Michaud, L.; Auray-Blais, C. Improved ways to screen for patients with fabry disease, involving optometry in a multidisciplinary approach. Can. J. Optom. 2012, 74, 25-32. [CrossRef]

4. Lenders, M.; Stappers, F.; Niemietz, C.; Schmitz, B.; Boutin, M.; Ballmaier, P.J.; Zibert, A.; Schmidt, H.; Brand, S.M.; Auray-Blais, C.; et al. Mutation-specific fabry disease patient-derived cell model to evaluate the amenability to chaperone therapy. J. Med. Genet. 2019, 56, 548-556. [CrossRef] [PubMed]

5. Provençal, P.; Boutin, M.; Dworski, S.; Au, B.; Medin, J.A.; Auray-Blais, C. Relative distribution of Gb3 isoforms/analogs in NOD/SCID/fabry mice tissues determined by tandem mass spectrometry. Bioanalysis 2016, 8, 1793-1807. [CrossRef]

6. Boutin, M.; Menkovic, I.; Martineau, T.; Vaillancourt-Lavigueur, V.; Toupin, A.; Auray-Blais, C. Separation and analysis of Lactosylceramide, Galabiosylceramide, and Globotriaosylceramide by LC-MS/MS in urine of fabry disease patients. Anal. Chem. 2017, 89, 13382-13390. [CrossRef] [PubMed]

7. Abaoui, M.; Boutin, M.; Lavoie, P.; Auray-Blais, C. tandem mass spectrometry multiplex analysis of methylated and non-methylated urinary Gb3 isoforms in fabry disease patients. Clin. Chim. Acta 2016, 452, 191-198. [CrossRef]

8. Boutin, M.; Auray-Blais, C. Multiplex tandem mass spectrometry analysis of novel plasma lyso-Gb3-related analogues in fabry disease. Anal. Chem. 2014, 86, 3476-3483. [CrossRef]

9. Lavoie, P.; Boutin, M.; Auray-Blais, C. Multiplex analysis of novel urinary lyso-Gb3-related biomarkers for fabry disease by tandem mass spectrometry. Anal. Chem. 2013, 85, 1743-1752. [CrossRef]

10. Auray-Blais, C.; Boutin, M.; Gagnon, R.; Dupont, F.O.; Lavoie, P.; Clarke, J.T.R. Urinary globotriaosylsphingosine-related biomarkers for fabry disease targeted by metabolomics. Anal. Chem. 2012, 84, 2745-2753. [CrossRef]

11. Dupont, F.O.; Gagnon, R.; Boutin, M.; Auray-Blais, C. A Metabolomic study reveals novel plasma lyso-gb3 analogs as fabry disease biomarkers. Curr. Med. Chem. 2012, 20, 280-288. [CrossRef]

12. Boutin, M.; Auray-Blais, C. Metabolomic discovery of novel urinary Galabiosylceramide analogs as fabry disease biomarkers. J. Am. Soc. Mass Spectrom. 2015, 26, 499-510. [CrossRef] [PubMed]

13. Manwaring, V.; Boutin, M.; Auray-Blais, C. A metabolomic study to identify new Globotriaosylceramide-related biomarkers in the plasma of fabry disease patients. Anal. Chem. 2013, 85, 9039-9048. [CrossRef]

14. Auray-Blais, C.; Blais, C.M.; Ramaswami, U.; Boutin, M.; Germain, D.P.; Dyack, S.; Bodamer, O.; Pintos-Morell, G.; Clarke, J.T.; Bichet, D.G.; et al. Urinary biomarker investigation in children with fabry disease using tandem mass spectrometry. Clin. Chim. Acta 2015, 438, 195-204. [CrossRef] [PubMed]

15. Auray-Blais, C.; Lavoie, P.; Boutin, M.; Ntwari, A.; Hsu, T.R.; Huang, C.K.; Niu, D.M. Biomarkers associated with clinical manifestations in fabry disease patients with a late-onset cardiac variant mutation. Clin. Chim. Acta 2017, 466, 185-193. [CrossRef] [PubMed]

16. Nowak, A.; Huynh-Do, U.; Krayenbuehl, P.A.; Beuschlein, F.; Schiffmann, R.; Barbey, F. Fabry disease genotype, phenotype, and migalastat amenability: Insights from a national cohort. J. Inherit. Metab. Dis. 2020, 43, 326-333. [CrossRef]

17. Germain, D.P.; Elliott, P.M.; Falissard, B.; Fomin, V.V.; Hilz, M.J.; Jovanovic, A.; Kantola, I.; Linhart, A.; Mignani, R.; Namdar, M.; et al. The effect of enzyme replacement therapy on clinical outcomes in male patients with fabry disease: A systematic literature review by a European panel of experts. Mol. Genet. Metab. Rep. 2019, 19, 100454. [CrossRef]

18. Medin, J.A. FACTS fabry gene therapy clinical trial: Two year data. Mol. Genet. Metab. 2019, 126, S7-S16. [CrossRef]

19. Wagner, B.D.; Accurso, F.J.; Laguna, T.A. The applicability of urinary creatinine as a method of specimen normalization in the cystic fibrosis population. J. Cyst. Fibros. 2010, 9, 212-216. [CrossRef]

20. Schiffmann, R.; Waldek, S.; Benigni, A.; Auray-Blais, C. Biomarkers of fabry disease nephropathy. Clin. J. Am. Soc. Nephrol. 2010, 5, 360-364. [CrossRef]

21. Mills, K.; Morris, P.; Lee, P.; Vellodi, A.; Waldek, S.; Young, E.; Winchester, B. Measurement of urinary $\mathrm{CDH}$ and $\mathrm{CTH}$ by tandem mass spectrometry in patients hemizygous and heterozygous for Fabry disease. J. Inherit. Metab. Dis. 2005, 28, 35-48. [CrossRef] [PubMed]

22. Whitfield, P.D.; Calvin, J.; Hogg, S.; O’Driscoll, E.; Halsall, D.; Burling, K.; Maguire, G.; Wright, N.; Cox, T.M.; Meikle, P.J.; et al. Monitoring enzyme replacement therapy in Fabry disease-Role of urine globotriaosylceramide. J. Inherit. Metab. Dis. 2005, 28, 21-33. [CrossRef] [PubMed] 
23. Auray-Blais, C.; Lavoie, P.; Boutin, M.; Abaoui, M. High-risk screening for fabry disease: Analysis by tandem mass spectrometry of Globotriaosylceramide (Gb3) in urine collected on filter paper. Curr. Protoc. Hum. Genet. 2017, 93, 17.26.1-17.26.12. [CrossRef] [PubMed]

24. Auray-Blais, C.; Cyr, D.; Ntwari, A.; West, M.L.; Cox-Brinkman, J.; Bichet, D.G.; Germain, D.P.; Laframboise, R.; Melançon, S.B.; Stockley, T.; et al. Urinary Globotriaosylceramide excretion correlates with the genotype in children and adults with fabry disease. Mol. Genet. Metab. 2008, 93, 331-340. [CrossRef]

25. Lavoie, P.; Boutin, M.; Abaoui, M.; Auray-Blais, C. Fabry disease biomarkers: Analysis of urinary Lyso-Gb3 and seven related analogs using tandem mass spectrometry. Curr. Protoc. Hum. Genet. 2016, 90, 17.22.1-17.22.12. [CrossRef]

26. Aickin, M.; Gensler, H. Adjusting for multiple testing when reporting research results: The bonferroni vs holm methods. Am. J. Public Health 1996, 86, 726-728. [CrossRef]

(C) 2020 by the authors. Licensee MDPI, Basel, Switzerland. This article is an open access article distributed under the terms and conditions of the Creative Commons Attribution (CC BY) license (http://creativecommons.org/licenses/by/4.0/). 